\title{
An Advanced Wireless System for Emergency Management in Hospitals
}

\author{
Angelo Biscotti ${ }^{1}$, Sara Spadoni ${ }^{1}$, Berardo Naticchia ${ }^{1}$ and Alessandro Carbonari ${ }^{2}$ \\ 1Polytechnic University of Marche, Smart Space Solutions SRL, Via Brecce Bianche, 60131 Ancona, Italy \\ 2Polytechnic University of Marche, DACS Department, Engineering Faculty, Ancona, Italy. Tel: \\ +390712204397; Fax: +39 0712204582; Mobile: +39 3402502768, alessandro.carbonari@univpm.it
}

\begin{abstract}
This paper reports preliminary results achieved during the experimentation of a new real time localization system, especially designed in support of hospitals' emergency management. This system represents the first step of a wider ongoing research project, carried on by the authors in collaboration with the academic spinoff company Smart Space Solutions SRL, aimed at the developing of the W.E.M.A.S. (Wireless Emergency Management Aiding System) system, whose final aim is increasing hospitals' efficiency in the management of the maxi emergencies consequent to disasters. WEMAS uses advanced wireless localization tracking and communication technologies to provide a number of features, such as: patient's flows and patient's healthcare progress tracking, equipment and medical personnel tracking, triage information management, ambulance scheduling and early communication of patient status from ambulance to the receiving emergency division. As a side effect, WEMAS system will equip hospitals with this new network infrastructure to optimize patient logistics for routine management. This paper describes also tests performed on the first release of WEMAS system, in the Emergency Division of the Hospital of Senigallia (Italy), demonstrating its features and technological feasibility.
\end{abstract}

Keywords: RTLS; wireless communication; disaster response; emergency management.

\section{Introduction}

This paper concerns the first step of a wider ongoing research project, named WEMAS (Wireless Emergency Management Aiding System), and carried out by the Department of Architecture Constructions and Structures (DACS) of the Polytechnic University of Marche, the academic spin-off company Smart Space Solutions SRL and the hospital of Senigallia.

The WEMAS, that will be developed to increase hospitals' emergency divisions efficiency in the management of maxi-emergencies consequent to disasters, in its final release will provide the following functionalities: support to first aid management on disaster fields; support to patient transfer from disaster field to hospital; support to emergency division activities within hospital.

As a consequence, the WEMAS will have positive implications also in hospitals' daily activities, allowing patients' healthcare flow tracking, optimization of resource management and also facility tracking to prevent loss or robbery attempts.

The project is compliant to the Marche Region's legislation that, by means of the regional act "DGR 49/2004", imposes that every hospital must arrange a PEIMAF plan ("Piano di Emergenza Massiccio Afflusso Feriti") devoted to coordination of hospital's activities in case of massive victim afflux consequent to disasters.

Nevertheless, empirical experience has attested the inadequacy of the PEIMAF plan in maxi-emergencies management, because of the adoption of traditional communication and management instruments. Even the most recent research, carried out in countries very sensitive to catastrophic events, like Japan or USA, realized inadequacy of traditional means when coping with catastrophic events (Wickramasinghe et al. 2006). The last catastrophic event is represented by September the 11th attacks, when inefficient communication between disaster field and neighbor hospitals was experienced.

Nowadays, limits could be fortunately overcame by the advent of recent IC technologies, providing a valid support in hospital's activities management (Chao et al. 2007), like faster patient's identification, patients' healthcare progress real-time tracking and so on. Some examples of such systems have been developed, tested and adopted in hospitals, but they all tend to be very invasive, moreover when installed in existing buildings. 
This paper presents first studies, aimed at developing a first WEMAS system release for emergency divisions, carried out by the authors through the following steps:

- performance analyses to discern the system's main functionalities;

- first localization experimental tests, to perform the reliability of the system, through the use of signal quality indices (these tests have been carried out in one of the most difficult environments: the hospital of Senigallia).

The system will be based on ZigBee technology, as it provides such a tiny structure, considered critical for fast and reliable communication.

\section{State of the Art}

PEIMAF standard plans organize rescues operations on a three level structure: organization of a field hospital for first aid; victims transfer form disaster scene to hospital; victim management and care within hospital's divisions.

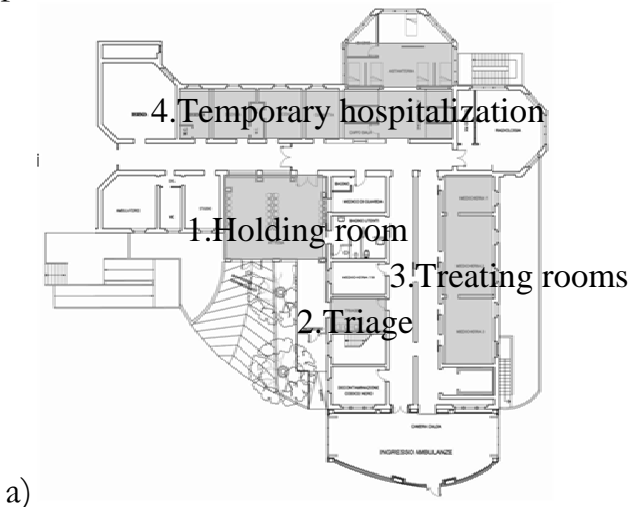

b)

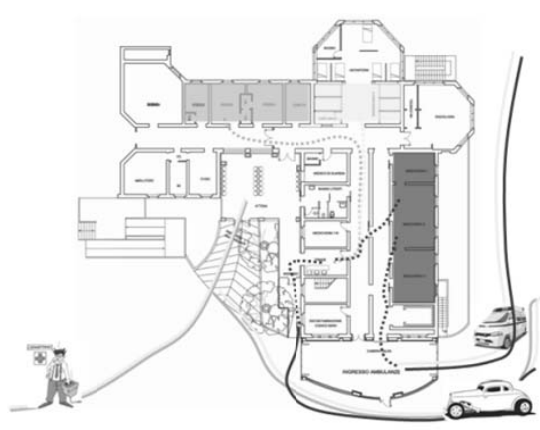

Figure 1. Senigallia Hospital Emergency Division organization in daily routine (a) and re-organization of victims paths in case of disaster management (b).

PEIMAF is applied just in case of massive patient flux and requires to fully re-organize rescue operations, from their triage to further medical care (Figure 1). As it can be noticed from comparing the two parts of Figure 1, in case of disaster Senigallia hospital emergency division occupies new areas, involving them in victims' care process. Figure 1-b also shows that victim's flows follow different courses in relation to their triage code. Due to the involvement of all hospital divisions, it requires a constant and continuous communication between personnel, not achievable through standard communication protocols.

The introduction of Radio Frequency Identification technologies could give a great impulse to the development of innovative real-time management systems, applied at different complex operative environments like hospitals. These systems, based upon various communication protocols (WiFi, IRDA, RFID, UWB) have been installed and employed in few hospitals. However they all present the same limits, such as:

1) high power consumption, requiring connection to the electric system;

2) invasive installations of big sized devices and cabling.

For that reason, the WEMAS project wants to release a new product.

\section{The New Approach Brought about by Our W.E.M.A.S.}

Table 1 illustrates the results of analyses carried out together with the hospital of Senigallia's personnel, relative to all the functionalities that could be reasonably provided by the WEMAS for automating hospital practices. For instance, check-in would change it standard procedures (assignment of a paper form to every patient containing his/her ID, triage code and health status), in favor of a badge to be automatically updated during patient's healthcare progress. The main limit of the standard practice lays in the frailty of paper documents and risks of document loss.

Instead, according to the WEMAS, patient's ID and data will be recorded in the central hospital database through a local computer unit or a PDA, passing through emergency division's intra-network. The same ID will be also associated to a bracelet worn by the patient and containing a Zigbee End-Device dispositive, able 
to communicate with portable and fixed wireless devices of a network installed in the hospital. Portable devices will also be provided to doctors, nurses and hospital assets, allowing for real time monitoring of patients and healthcare resources' positions.

Table 1. The WEMAS' features foreseen for the final release.

\begin{tabular}{|c|c|}
\hline Query & Check out \\
\hline - Patient's identification & - Admission \\
- Healthcare plan & - Dimission \\
- Clinical parameters & - Self-dimission \\
- Real-time location & Health care \\
\cline { 2 - 2 } - Timeling & \multicolumn{2}{|c|}{ Triage code assignation } \\
- Available resource & Request \\
\hline Alert & - Medical expertise scouting and consulting \\
\hline - Patient's support call & - Medical test or instrument reservation \\
- Patient's clinical parameter monitoring & - Busy \\
- Patient's drift & - Call monitoring \\
- Hobbery attempts & \\
\hline
\end{tabular}

In addition, by means of their PDAs, doctors will be able to visualize patient's identity and his healthcare progress, by connecting to patients' bracelets. Through their devices they could also ask for a medical expertise scouting and consulting, as shown in Figure 3.

a)

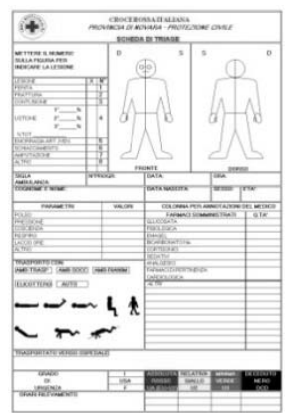

b)

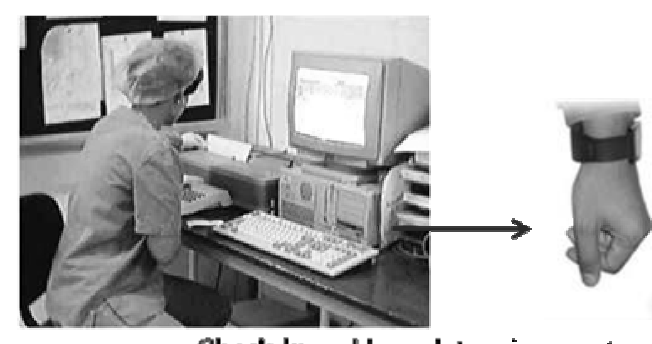

Check-ln mnd braceler assigument

Figure 2. Traditional triage paper (a); Programmation and Assignation of patient's identification bracelet (b).
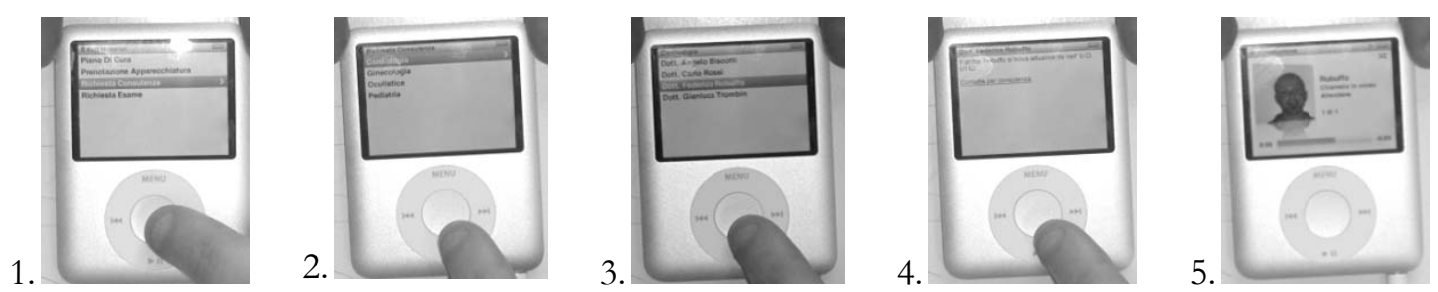

Figure 3. The procedure for "Medical expertise scouting and consulting" through medical PDAs

\section{The WEMAS Technology}

The WEMAS provides its features through hardware and software components based on the IEEE 802.15.4 standard medium access and Zigbee stack communication protocol. These protocols are intended for use in embedded applications requiring low data rates and low power consumption, and present a lot of useful properties to be implemented in health care workplaces (Lorincz et al. 2006).

ZigBee's current focus is to define a general-purpose, cheap, self-organizing mesh network. There are several advantages provided by the Zigbee protocol, that make it strategic for hospital environments: it works in ISM band; it is reliable and self healing; it supports large number of nodes (16 bit address space); it is easy to deploy; it allows very long battery life; it is a safe and low cost technology. 
The WEMAS exploits three Zigbee device types (Figure 4-a), compliant to standard application profile: each one presenting specific features, as depicted in Table 2.

Our WEMAS devices are equipped with the integrated radio and microcontroller Chipcon/Texas Instruments CC2431 chip with 128K flash memory. CC2431 has an embedded localization hardware engine, which estimates the position of a node by evaluating the received signal strength (RSSI) attenuation (Michel et al. 2006), according to the Path Loss Model (PLM) formula:

$$
R S S I=-\left(A+10 \cdot n \cdot \log _{10}(d)\right)
$$

where $\mathrm{d}$ represents the distance in meters from the sender node, while $\mathrm{A}$ is the received signal in $\mathrm{dBm}$ at a distance of one meter with a sending power of $0 \mathrm{dbm}$.

The decentralized localization algorithm is performed entirely by the "blind" node, reducing the communication costs and bottlenecks. A mobile node who wants to evaluate its position sends a broadcast message to all ZRs of the network in its radio range; each ZR replies with a short message whose payload contains its fixed coordinates and the message's RSSI received, measured in dBm (see Figure 4-b). At least three RSSI indications are then written in the location hardware registers with ZRs coordinates, A and $n$ values of the PLM formula in eq. 1. Output contains the coordinates of the evaluated position. The procedure to evaluate $A$ and $n$ values is described in (Chipcon/Texas Instruments 2007).

Table 2. ZigBee device types with their features

\begin{tabular}{|c|c|c|}
\hline Zigbee Coordinator (ZC) & Zigbee Router (ZR) & $\begin{array}{r}\text { Zigbee Er } \\
\text { (ZF }\end{array}$ \\
\hline 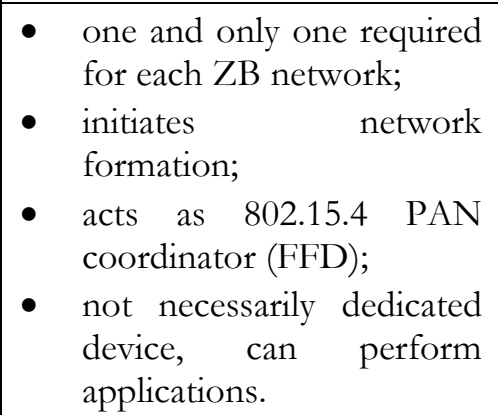 & 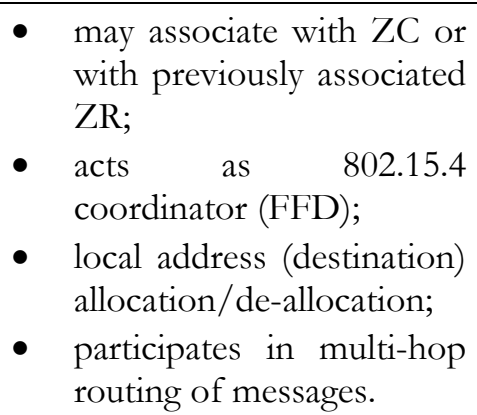 & $\begin{array}{l}\text { - } \text { mobile node; } \\
\text { - } \text { shall not allow } \\
\text { association; } \\
\text { - } \quad \text { shall not participate in } \\
\text { routing; } \\
\text { - } \quad \text { put to sleep by parents. }\end{array}$ \\
\hline
\end{tabular}

The engine is not capable to evaluate node's position if less than three ZRs reply to the broadcast request. In this case the position can be evaluate through gate control. The utilization of a dedicated hardware reduces considerably the delay of the mathematical process. The Zigbee standard profile suggests to have main powered ZR, in order to maintain the radio transceiver in listen mode and have the possibility of routing messages throughout the network. The necessity of using cables dramatically decreases the flexibility of the network in existent hospital environments, because fixed nodes have to be placed in proximity of an electrical source. However the Zigbee specifications allow to use a synchronous communication protocol which uses a special type of message called beacon.

The beacon is a sync message, which defines a time interval for message passing, but increases extremely the latency, in order to minimize duty cycle. WEMAS system uses a new approach, developed by the academic spin-off Smart Space Solutions srl. This proprietary protocol is intended for reduce ZR's duty cycle in asynchronous mode. It allows the arrangement of full wireless networks, because battery powered, requiring minimum maintenance efforts, with a 2 years long power life.

Each ZR node remains in sleep mode for reducing power consumption until it has to route a message or reply to a request. In these cases an asynchronous signal wakes it up from sleep and allows it to work without waiting for a sync. 


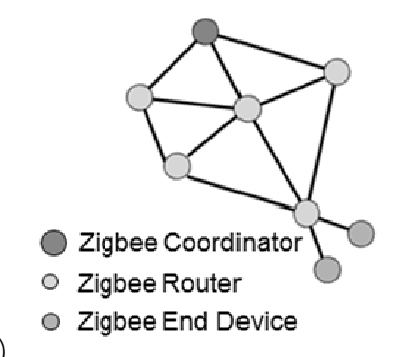

a)

Figure 4. example of a mesh Zigbee network (a); RSSI based message exchange for localization (b).

The localization hardware engine and the low power communication capabilities, combined with the selfhealing property, make the WEMAS extremely suitable for hospitals with respect to other tracking technologies.

RFID has a lower range, compared with costs of readers, and interference problems with existing LAN networks. Zigbee compliant devices instead aren't subject to significant interference with other wireless technologies. In (ZigBee Alliance 2007) it is showed how in addition to DSSS (direct sequence spread spectrum) technology, the 802.15.4 physical layer protocol increases the opportunities for coexistence by employing a technique, generally known as frequency division multiple access (FDMA). Even with the techniques described above, a ZigBee device may find itself sharing a channel with interferers. The approach taken by the IEEE in the 802.15.4 standard is known as carrier sense multiple access (CSMA). Both tests and everyday use in real environments with real data traffic bear prove ZigBee's robustness.

Infrared systems need to have a line of sight to communicate one another and have not complex communication capabilities, so it can be used just for gate control. Instead Zigbee devices do not suffers multipath effects, thanks to application of mesh networking. Since multiple paths exist in a mesh network, messages can be sent into several directions. If one path is lost for some reasons or the link budget of a connection is too low, the message is likely to direct it along another path.

UWB allows very accurate positioning, but it is very expensive and complex.

Wi-Fi boasts a higher range, but the infrastructure is expensive and needs electric power. The signal strength of a Zigbee device is proportional to the transmission power and decreases in presence of obstructions such as doors or walls (Figure 5).

Some environments need to increase the number of ZR to maintain redundancy and avoid bottlenecks.

\begin{tabular}{|l|c|c|c|c|c|c|}
\hline \multirow{2}{*}{ Factor } & \multicolumn{2}{c|}{$\mathbf{4 3 3} \mathbf{M H z}$} & \multicolumn{2}{c|}{$\mathbf{8 6 8} \mathbf{M H z}$} & $\mathbf{2 . 4} \mathbf{G H z}$ \\
\cline { 2 - 7 } & Loss & Attenuation & Loss & Attenuation & Loss & Attenuation \\
\hline Open office & $0 \%$ & $0 \mathrm{~dB}$ & $0 \%$ & $0 \mathrm{~dB}$ & $0 \%$ & $0 \mathrm{~dB}$ \\
\hline Window & $<5 \%$ & $<1 \mathrm{~dB}$ & $15 \%$ & $1-2 \mathrm{~dB}$ & $30 \%$ & $3 \mathrm{~dB}$ \\
\hline Thin wall (plaster) & $25 \%$ & $3 \mathrm{~dB}$ & $35 \%$ & $3-4 \mathrm{~dB}$ & $50 \%$ & $5-8 \mathrm{~dB}$ \\
\hline Medium wall (wood) & $40 \%$ & $4-6 \mathrm{~dB}$ & $50 \%$ & $5-8 \mathrm{~dB}$ & $70 \%$ & $10-12 \mathrm{~dB}$ \\
\hline Thick wall (concrete) & $50 \%$ & $5-8 \mathrm{~dB}$ & $60 \%$ & $9-11 \mathrm{~dB}$ & $85 \%$ & $15-20 \mathrm{~dB}$ \\
\hline Armoured wall (reinforced concrete) & $70 \%$ & $10-12 \mathrm{~dB}$ & $80 \%$ & $12-15 \mathrm{~dB}$ & $90 \%$ & $20-25 \mathrm{~dB}$ \\
\hline Floor or ceiling & $50 \%$ & $5-8 \mathrm{~dB}$ & $60 \%$ & $9-11 \mathrm{~dB}$ & $85 \%$ & $15-20 \mathrm{~dB}$ \\
\hline Armoured floor or ceiling & $70 \%$ & $10-12 \mathrm{~dB}$ & $80 \%$ & $12-15 \mathrm{~dB}$ & $90 \%$ & $20-25 \mathrm{~dB}$ \\
\hline Rain and/or Fog & $90 \%$ & $20-25 \mathrm{~dB}$ & $95 \%$ & $25-30 \mathrm{~dB}$ & $?$ ?? & $? *$ \\
\hline
\end{tabular}

Figure 5. Signal attenuation in correspondence of different kinds of obstructions (OneRF Technology, 2007).

\section{Preliminary Experiments on the Wemas Real-time Localization System}

As previously explained, in order to assure that all the WEMAS' features will work properly, it is necessary to develop a reliable wireless sensor network (i.e. reliable communication among all the nodes that is measured through RSSI received by every ZR within radio range).

Preliminary tests were performed in the worst environment where WEMAS could be likely installed, that is to say the emergency division of the Senigallia hospital, built with a heavy masonry structure (see Figure 6a), whose thickness ranges between 0.4 and $1.2 \mathrm{~m}$. 


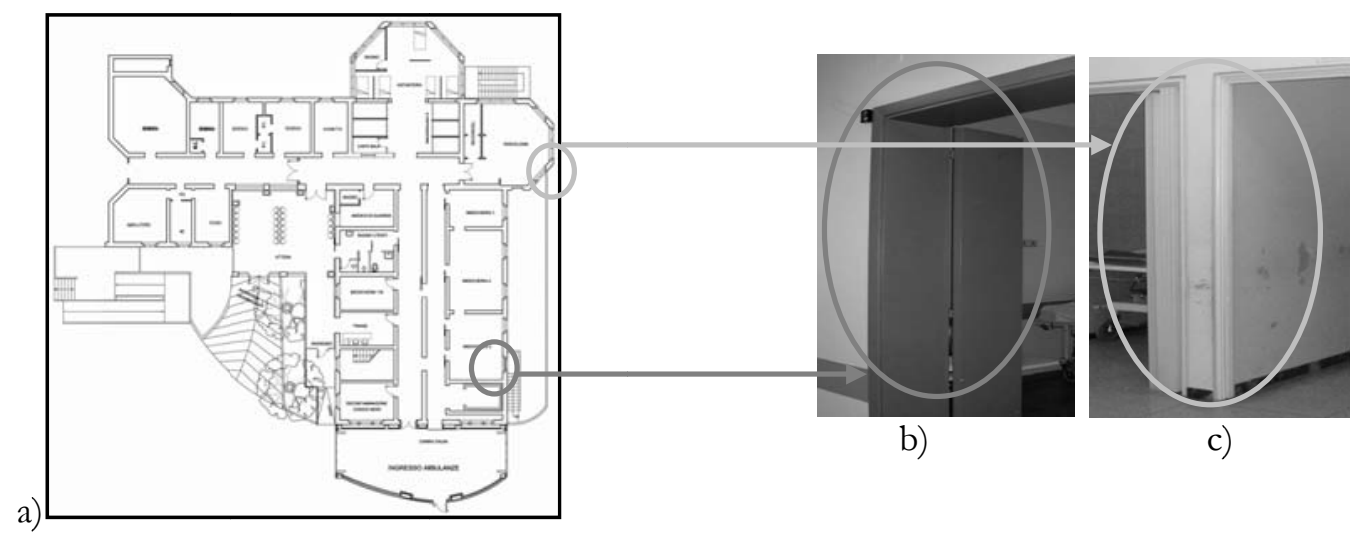

Figure 6. Ground level plan of the Senigallia Hospital Emergency division (a); details of a $0.4 \mathrm{~m}$ thich solid brick wall (b) and of a partitioning wall (c).

Senigallia Hospital Emergency Division is a three level building, characterized by very thick solid brick made walls (Figure 6-b), and partitioning walls built with hollow-solid bricks (Figure 6-c). Its cramped space organization makes localization a tougher task.

Following first preliminary experiments, the final testing have been carried out at the ground level of the Emergency Division. Figure 7 illustrates fixed devices positioning throughout the structure: as it can be noticed a ZC device has been placed close to the middle of the left side corridor, next to the left wall, while sixteen $Z R$ devices have been deployed as follows:

- nine ZR in the two corridors (no. 4, 5, 6, 7, 10, 12,13, 21, 22);

- six ZR in six different rooms along the corridors (no. 1, 14, 15, 16 17, 23);

- one ZR (no. 20) next to the door of one room.

The photographic report in Figure 7-b depicts some of the routers installed on the hospital's walls: it can be noticed that this suggested technology requires rather small sized devices, properly packaged and fixed on the walls with reversible means (like glue or similar systems).

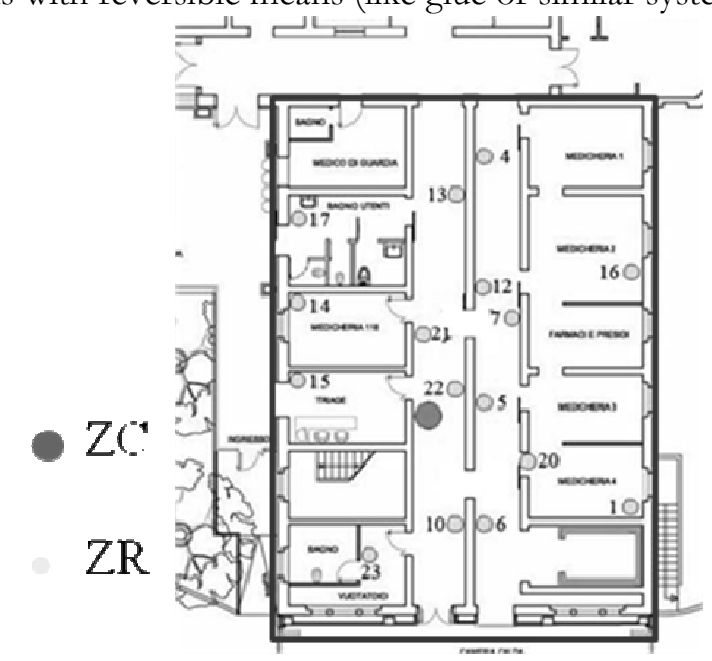

a)
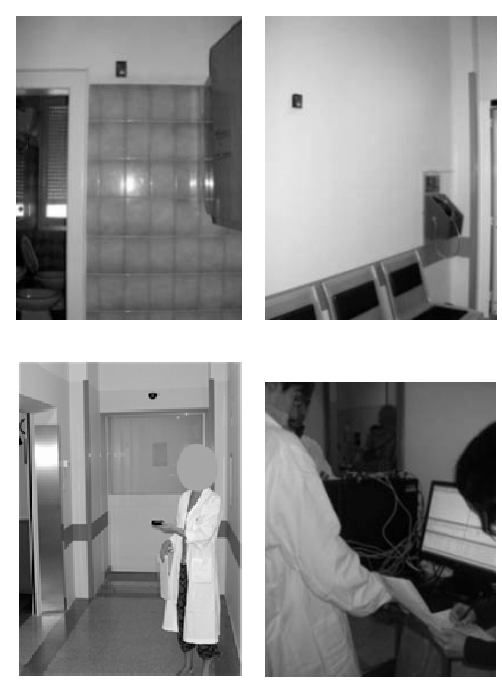

b)

Figure 7. WEMAS installation layout in Senigallia Hospital Emergency Division (a), and photographic report of some device positioning (b).

Instead ZED devices have been moved across nine different positions during testing, as illustrated in Figure 8 -a, in order to verify that all the room included within the emergency division can be tracked by our system. Localization have been performed using the algorithm detailed in paragraph 4.

It has to be outlined that our sensor devices hold a built-in mechanism for RSSI evaluation of the received signal always limited between $-95 \mathrm{dBm}$ and $-40 \mathrm{dBm}$ (that is to say, if the received RSSI is lower than $-95 \mathrm{dBm}$ than it is neglected; otherwise if it is higher than $-40 \mathrm{dBm}$, than it is outputted at $-40 \mathrm{dBm})$. A 
good communication quality is assured if RSSI value higher than $-85 \mathrm{dBm}$ is measured by at least one ZR, when the ZED is located in every position of the building.

Experimental tests gave back good results from this point of view. Figures 8-b and 8-c show some examples of the performed localization tests: in every case the received RSSI was higher than $-85 \mathrm{dBm}$, the strongest ZR was identified as the closest and a good match between the real and the estimated position for the blind node (i.e. ZED) was obtained.

As guide-line for further installations it was also noticed that: a $350 \mathrm{~m}^{2}$ large built area, surrounded and subdivided by heavy walls, can be monitored by using 16 ZRs, that is to say one ZR per $20 \mathrm{~m}^{2}$ large built area. ZRs must also be installed next to all door openings, in order to avoid ZED localization errors.

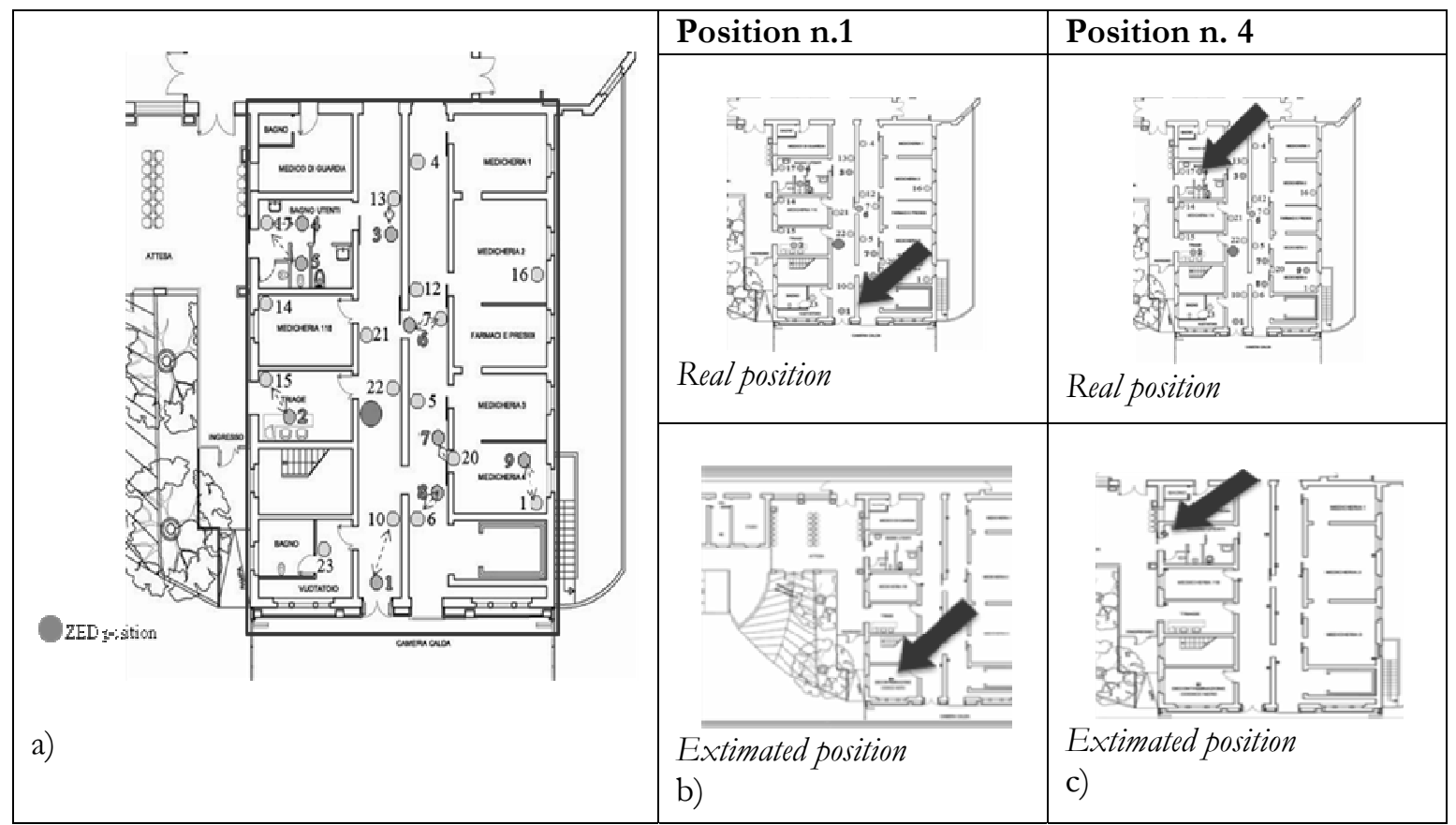

Figure 8. Various locations occupied by the ZED devices during testing (a); comparison between real and estimated positions (b, c).

\section{Conclusions}

Performance analyses relative to maxi-emergencies management allowed identifying a series of features that our WEMAS can offer in order to become a strategic instrument either in the case of massif victims affluence, or in hospital's daily activities. In the last case, the adoption of the WEMAS can be useful for optimized resource and communication management, besides improving structure's safety level, achievable through the installation of the full-wireless, low invasive system, based on our unique technology.

First localization experiments demonstrated the reliability of the WEMAS, even in case of buildings having very heavy structures.

Within little time, a prototypal version of WEMAS system, endowed with some further functionalities of Table 1, will be installed and tested in the emergency Department of Senigallia Hospital for continuous experimental monitoring.

\section{References}

[1] C. Chao, W. Chen, Y. Chy, B. Lin (20079, "Improving patient safety with RFID and MOBILE technology", Int J Electron Healthc, 3(2): 175-92

[2] Chipcon / Texas Instruments (2007), "System-on-Chip for 2.4 GHz ZigBee®/ IEEE 802.15.4 with Location Engine" <URL:http:// focus.ti.com/lit/ds/symlink/cc2431.pdf>

[3] K. Lorincz, M. Welsh (2006), "MoteTrack: a robust, decentralized approach to RF-based location tracking", Pres Ubiquit comput., Springer-Verlag London Limited, DOI 10.1007/s00779-006-0095-2. 
[4] D. Michel, D. Toggenburger (2006), "Sensor network soccer", Diploma Thesis WS, University of Applied Sciences Rapperswil HSR, Department of Electrical Engineering, Institute for Communication Systems.

[5] OneRF Technology (2007), "B2400MC-uTiny Technical Manual" <URL:http://backoffice.inware.it/files/prodotti/download/onerf/manuale\%20zigbee\%201.pdf>

[6] N. Wickramasinghe, R.K. Bali, R.G. Naquib (2006), "Application of knowledge management and intelligent continuum for medical emergencies and disaster scenarios", Conf. Proc. IEEE "Eng Med Biol Soc.", 1:5149-52

[7] Zigbee Alliance White Paper (2007), "ZigBee and Wireless Frequency Coexistence", < www.zigbee.org/imwp/download.asp? ContentID=11745> 